\title{
Soluzione per il controllo dei composti farmaceutici nelle acque reflue
}

\author{
Antonia Marinelli ${ }^{1}$ \\ ${ }^{1}$ University of Salerno
}

\begin{abstract}
Negli ultimi tempi, soprattutto in seguito alla diffusione del Covid-19, si è affermata l'importanza del trattamento terziario delle acque reflue a causa della persistenza dei prodotti farmaceutici e dei virus nelle acque anche dopo i trattamenti.

Una possibile soluzione per il risanamento delle acque reflue potrebbe essere l'applicazione della sonolisi, uno dei processi avanzati di ossidazione.

La sonolisi è una scissione molecolare generata dall'energia prodotta dagli ultrasuoni, che hanno lo scopo di decontaminare le acque da inquinanti chimici tramite l'induzione di fenomeni cavitativi.

In letteratura è dimostrata l'efficienza di questo trattamento terziario su singoli prodotti farmaceutici, ma mancano totalmente studi riguardo l'efficacia su miscele.

Al fine di ottenere i migliori risultati, si è studiato come il processo ad ultrasuoni agisca sulla degradazione di prodotti farmaceutici in singole soluzioni o in miscele addizionate nelle acque reflue urbane, al variare della densità di potenza, della concentrazione iniziale del substrato, del pH della soluzione iniziale e con l'insufflaggio d'aria.

È stato scoperto che la conversione di prodotti farmaceutici è migliorata con la maggiore densità di potenza applicata, in condizioni acide e in presenza di aria disciolta.
\end{abstract}




\section{Il problema dell'inquinamento delle acque reflue con prodotti}

\section{farmaceutici}

La diffusa presenza di prodotti farmaceutici e per la cura della persona negli ambienti acquatici è un problema universalmente riconosciuto le cui conseguenze restano ad oggi sconosciute. ${ }^{1,2,3,4,5}$

Il problema della scarsità di acqua dolce ha indotto il ministero a individuare come soluzione il riutilizzo delle acque reflue. La normativa italiana, il D.M. 93/06 e la direttiva Europea 91/271/CEE definiscono i requisiti minimi di qualità relativi a diversi parametri micro-biologici e chimico-fisici per il riutilizzo delle acque reflue per l'uso irriguo, civile ed industriale.

Purtroppo non prevede parametri o raccomandazioni che richiamino l'attenzione delle autorità sanitarie sui farmaci come contaminanti.

Uno studio abbastanza recente ha dimostrato come questi cocktail farmaceutici presenti nelle acque reflue trattate, che vengono poi riutilizzate per l'irrigazione dei campi, siano in grado di indurre stress alle piante. ${ }^{6}$

Si comprende quindi l'importanza del trattamento terziario delle acque reflue.

Le fonti responsabili della presenza di prodotti farmaceutici nelle acque possono essere gli impianti di trattamento delle acque reflue, gli ospedali, le acque reflue domestiche non trattate o alcune fonti sconosciute. Tra questi di notevole importanza sicuramente gli effluenti degli impianti di trattamento delle acque reflue urbane.

I farmaci più frequentemente rilevati nell'ambiente acquatico sono:

- $\quad$ Diclofenac (DCF), un agente non steorideo antinfiammatorio;

- $\quad$ Amoxicillina (AMX) un antibiotico $\beta$-lattamico a spettro moderato;

- Carbamazepina (CBZ), un farmaco anticonvulsionante e stabilizzante dell'umore. 
Diversi studi recenti hanno rivelato che questi composti, soprattutto se in miscela, sono difficili da rimuovere dalle acque con le fasi convenzionali di trattamento di purificazione e, proprio per questo, di recente si sono cercati metodi non biologici per la loro rimozione dalle acque.

Molteplici sono i fattori che intervengono nella selezione della tecnologia più appropriata di trattamento delle acque reflue urbane quali ad esempio, la necessità di recupero dei nutrienti, l'efficienza energetica, la necessità di riutilizzo dell' acqua e dei fanghi. ${ }^{7}$

Una delle tecnologie più innovative è l'applicazione dell'irradiazione ultrasonica per il trattamento di contaminanti nell'acqua.

Il meccanismo di funzionamento della sonicazione ha come principio l'utilizzo gli ultrasuoni, che sono suoni con frequenza superiore a $20 \mathrm{kHz}$. Questi suoni innescano il fenomeno della cavitazione, con formazione, crescita e successivo collasso di microbolle, o cavità che si verificano in intervalli di tempo estremamente piccoli rilasciando grandi quantità di energia. ${ }^{8}$

L'efficacia del trattamento dipende dal tipo di farmaco trattato, dalla densità di potenza delle onde ultrasoniche, dalle concentrazioni iniziali del substrato, dal pH iniziale e dall'insufflaggio d'aria. Applicazioni recenti hanno dimostrato che il processo ad ultrasuoni può essere applicato con successo nella rimozione di prodotti farmaceutici e per la cura della persona come il triclosan, l'ossido di trifenilfosfina ${ }^{9}$, l'ibuprofene $^{10}$ e diflonac ${ }^{11}$.

Tuttavia, in letteratura non risultano studi sulla degradazione sonochimica di questi prodotti in miscela. ${ }^{12}$ 


\section{L'applicazione degli ultrasuoni sulle miscele di prodotti farma-}

\section{ceutici}

Per comprendere meglio come il processo ad ultrasuoni possa essere applicato nella degradazione di miscele di componenti farmaceutici si fa riferimento allo studio condotto da Naddeo et al., 2009 il cui obiettivo è stato quello di comprendere quali siano le condizioni migliori affinché le radiazioni a ultrasuoni a bassa frequenza $(20 \mathrm{kHz})$ risultino efficaci sulle miscele dei tre prodotti farmaceutici più frequentemente rilevati negli ambienti acquatici (DCF, AMX, CBZ). ${ }^{12}$

I campioni necessari per lo studio sono stati prelevati da un piccolo impianto di trattamento delle acque reflue progettato per 30.000 abitanti equivalenti, in cui confluivano esclusivamente acque domestiche . Durante il campionamento l'impianto era in funzione con una portata giornaliera media di $7000 \mathrm{~m}^{3}$ al giorno. La caratterizzazione dei campioni con relativi parametri sono riportati nella tabella di seguito.

Una volta prelevati e trasferiti in laboratorio, ai campioni sono stati addizionati i prodotti farmaceutici in tre diversi tipi di miscela, in modo da disporre di tre livelli di tossicità, a seconda dei valori iniziali di carbonio organico totale (TOC):

- $\quad$ Elevata tossicità (M1): 10 mg/L per AMX, 5mg/L per CBZ e 2.5 mg/L per DCF

- $\quad$ Media tossicità (M2): 5mg/L per AMX, $2.5 \mathrm{mg} / \mathrm{L}$ per CBZ e $2.5 \mathrm{mg} / \mathrm{L}$ per DCF

- $\quad$ Bassa tossicità (M3): 2.5 mg/L per AMX, 2.5 mg/L per CBZ e 2.5 mg/L per DCF

La concentrazione di DCF è costante, perché non è stato osservato alcun effetto tossico relativo allo stesso in campioni di acque reflue trattate e non trattate. ${ }^{13}$

Il campione, dopo essere stato riversato in una nave cilindrica, opportunamente addizionato alla 
miscela, è stato bombardato con un generatore di ultrasuoni dotato di tromba al titanio.

Gli ultrasuoni hanno innescato il processo di cavitazione, che consiste nella formazione di piccole bolle nel mezzo liquido e il loro successivo collasso.

La cavitazione ha due effetti la degradazione di inquinanti e la produzione di radicali liberi.

Purtroppo, come già osservato in altri studi ${ }^{14}$, poiché questi prodotti farmaceutici sono solubili in acqua e non volatili, la degradazione all'interno della bolla di cavitazione dovrebbe essere insignificante e, quindi, l'unico meccanismo di degradazione efficace sui prodotti farmaceutici sono le reazioni indotte dai radicali idrossilici. ${ }^{15}$

Per monitorare la concentrazione dei farmaci durante l'esperimento e la concentrazione di ciascun farmaco, tramite il picco caratteristico dell'assorbanza ,si è utilizzato uno spettrofotometro UV .

L'obiettivo è stato quello di valutare l'ecotossicità del campione prima, durante e dopo il trattamento, al variare della concentrazione iniziale del substrato, della potenza applicata, del pH della soluzione, dell'effetto dello sparging d'aria, e della formazione di $\mathrm{H}_{2} \mathrm{O}_{2}$.

Per conoscere l'ecotossicità sono stati utilizzati un crostaceo il Daphnia magna, una microalga la pseudokirchneriella subcapitata, e un crescione inglese (pianta) il lepidium sativum, prima e dopo il trattamento senza diluire i campioni. ${ }^{12}$

I parametri presi in considerazione per monitorare la tossicità sono il TOC, la domanda chimica di ossigeno (COD) e quella biochimica di ossigeno $\left(\mathrm{BOD}_{5}\right)$.

Si è compreso che per i singoli prodotti il tasso di degradazione aumenta linearmente con le concentrazioni iniziali di TOC, invece, per le miscele il tasso risulta indipendente dalla concentrazione iniziale di TOC.

Presi singolarmente né AMX né CBZ si sono rivelati tossici per P. subcapitata quanto la miscela M1 addizionata prima della sonicazione a dimostrazione del fatto che la tossicità è sinergica in 
caso di miscele.

Per P. Subcapitata si è osservato che:

- Per la miscela M1 sebbene si sia verificato uno spostamento della biodegradabilità da 30 a 60 minuti, ciò non era sufficiente per rimuovere la tossicità dei sottoprodotti recalcitranti ed è stata ottenuta una superiore tossicità rispetto a quella del campione WW non trattato.

- Per la miscela M2 non si sono verificati né un aumento né una diminuzione di tossicità.

- Per la miscela M3 la tossicità è stata efficacemente rimossa tramite la sonicazione.

Per quanto riguarda la D. Magna solo la miscela M3 ha mostrato una lieve tossicità (immobilizzazione del 20\%) prima e dopo sonificazione .

La tossicità dei campioni per L. sativum (mediante indice di germinazione, indice GL,\%), valutata per la prima volta in questo studio, è diminuita dopo aver addizionato il campione con le miscele dei farmaci ${ }^{12}$.

Lo studio ha dimostrato come i prodotti farmaceutici in miscela agiscano sinergicamente nell'aumentare la tossicità delle acque reflue.

L'utilizzo della sonicazione a bassa frequenza si è rivelato efficace sia sulle miscele che sui singoli farmaci, soprattutto con la maggiore densità di potenza applicata, in condizioni acide e in presenza di aria disciolta, anche se la tossicità su P. Subcapitata è aumentata.

\section{Possibili sviluppi futuri}

L'obiettivo principale del lavoro è stato cercare le migliori condizioni per effettuare una depurazione efficace delle acque reflue al fine di consentire il riutilizzo delle stesse in totale sicurezza.

L'applicazione della sonicazione a bassa frequenza, tuttavia, si è rivelata efficace solo per miscele a media e bassa tossicità, rivelandosi inutile, se non addirittura dannosa, per le miscele ad elevata 
tossicità.

Quindi, a mio parere, il trattamento ultrasonico è da utilizzare "con le pinze" e non può considerarsi una soluzione definitiva al problema dell'inquinamento delle acque a causa di prodotti farmaceutici.

Un ulteriore approfondimento andrebbe effettuato sui rischi, che i residui di questi cocktail farmaceutici, presenti nelle acque reflue, costituiscono per la salute umana e quelli legati all'aumento della resistenza dei batteri agli antibiotici.

Inoltre, la recente epidemia di Coronavirus evidenzia l'importanza della disinfezione per proteggere la salute pubblica.

Nella ricerca e nell'innovazione andrebbe studiato cos'accade quando si verifica la compresenza di prodotti farmaceutici con il Coronavirus nelle acque e quali rischi ci siano per la salute.

Infatti sebbene l'amministrazione statunitense per la sicurezza e la salute sul lavoro (OSHA) affermi che le attuali condizioni di disinfezione nei depuratori, come l'ossidazione con ipoclorito o acido peracetico e l'inattivazione mediante irradiazione ultravioletta (UV), dovrebbero essere sufficienti per proteggere i lavoratori delle acque reflue e la salute pubblica non ci sono ancora studi e conferme a riguardo.

Si afferma dunque l'importanza del trattamento terziario delle acque reflue al fine di garantire la sicurezza degli ambienti acquatici, tramite la collaborazione di più esperti provenienti da più settori ,per evitare danni alla salute pubblica.

\section{References}

1.Christensen, F. M. Pharmaceuticals in the Environment-A Human Risk?. Regulatory Toxicology and Pharmacology 28, 212-221 (1998). 
2.Halling-Sørensen, B. et al.. Occurrence fate and effects of pharmaceutical substances in the environment- A review. Chemosphere 36, 357-393 (1998).

3.Kolpin, D. W. et al. Pharmaceuticals Hormones, and Other Organic Wastewater Contaminants in U.S. Streams, 1999-2000: A National Reconnaissance. Environmental Science \& Technology 36, 1202-1211 (2002).

4.Heberer, T. Occurrence fate, and removal of pharmaceutical residues in the aquatic environment: a review of recent research data. Toxicology Letters 131, 5-17 (2002).

5.Kasprzyk-Hordern, B., Dinsdale, R. M. \& Guwy, A. J. The occurrence of pharmaceuticals personal care products, endocrine disruptors and illicit drugs in surface water in South Wales, UK. Water Research 42, 3498-3518 (2008).

6.Gorovits, R., Sobol, I., Akama, K., Chefetz, B. \& Czosnek, H. Pharmaceuticals in treated wastewater induce a stress response in tomato plants. Scientific Reports 10, (2020).

7.Kamble, S. J., Singh, A. \& Kharat, M. G. A hybrid life cycle assessment based fuzzy multicriteria decision making approach for evaluation and selection of an appropriate municipal wastewater treatment technology. Euro-Mediterranean Journal for Environmental Integration 2, (2017).

8.Gogate, P. R. Cavitation: an auxiliary technique in wastewater treatment schemes. Advances in Environmental Research 6, 335-358 (2002).

9.Emery, R. J., Papadaki, M., Santos, L. M. F. dos \& Mantzavinos, D. Extent of sonochemical degradation and change of toxicity of a pharmaceutical precursor (triphenylphosphine oxide) in water as a function of treatment conditions. Environment International 31, 207-211 (2005).

10.Méndez-Arriaga, F. et al.. Ultrasonic treatment of water contaminated with ibuprofen. Water Research 42, 4243-4248 (2008).

11.Naddeo, V., Belgiorno, V., Ricco, D. \& Kassinos, D. Degradation of diclofenac during sonolysis 
ozonation and their simultaneous application. Ultrasonics Sonochemistry 16, 790-794 (2009).

12.Naddeo, V., Meriç, S., Kassinos, D., Belgiorno, V. \& Guida, M. Fate of pharmaceuticals in contaminated urban wastewater effluent under ultrasonic irradiation. Water Research 43, 4019-4027 (2009).

13.Naddeo, V., Belgiorno, V., Kassinos, D., Mantzavinos, D. \& Meric, S. Ultrasonic degradation mineralization and detoxification of diclofenac in water: Optimization of operating parameters. Ultrasonics Sonochemistry 17, 179-185 (2010).

14.Zhang, Y., Geißen, S. \& Gal, C. Carbamazepine and diclofenac: Removal in wastewater treatment plants and occurrence in water bodies. Chemosphere 73, 1151-1161 (2008).

15.Hartmann, J. et al.. Degradation of the drug diclofenac in water by sonolysis in presence of catalysts. Chemosphere 70, 453-461 (2008). 


\section{Figure Captions}

Figure 1. Prodotti farmaceutici ( Unslpash )

Figure 2. Impianto di depurazione delle acque reflue (Unsplash)

Figure 3. Caratterizzazione delle acque reflue prima della contaminazione farmaceutica 


\section{Figures}

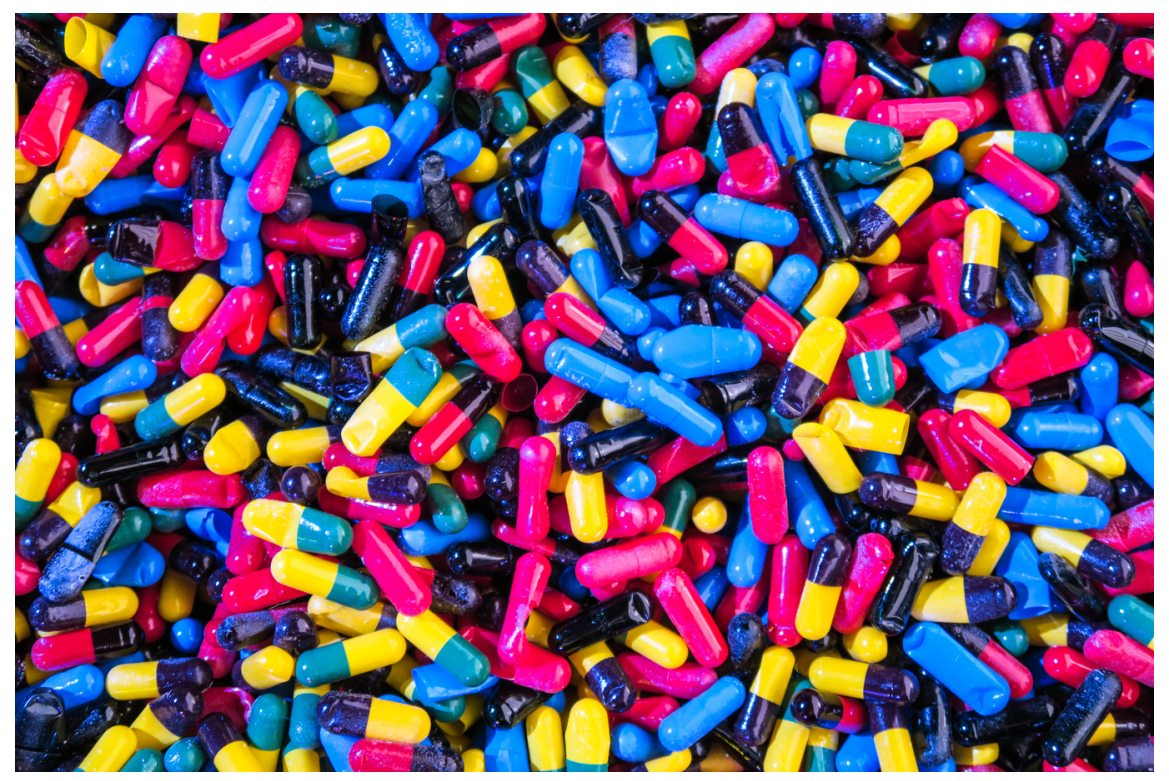

Figure 1: Prodotti farmaceutici ( Unslpash )

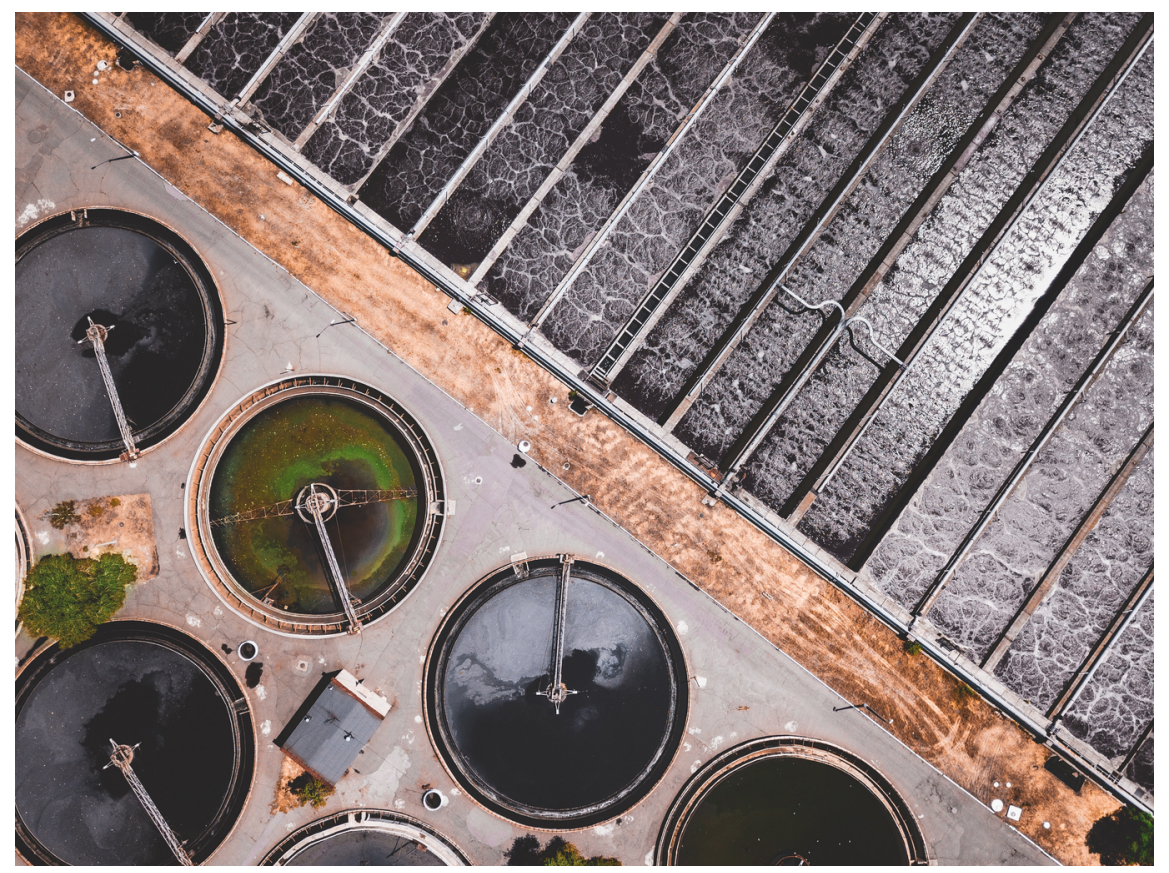

Figure 2: Impianto di depurazione delle acque reflue (Unsplash) 


\begin{tabular}{|c|c|c|}
\hline Parametri & Unità di misura & Valori \\
\hline Temperatura & ${ }^{\circ} \mathrm{C}$ & $20 \pm 2$ \\
\hline Conducibilità & $\mathrm{mScm}^{-1}$ & $2424 \pm 150$ \\
\hline ORP & $\mathrm{V}$ & $302 \pm 25$ \\
\hline DO & $\mathrm{ppm}$ & $5.35 \pm 0.7$ \\
\hline $\mathrm{pH}$ & - & $7.5 \pm 0.2$ \\
\hline Torbidità & NTU & $5 \pm 1$ \\
\hline TOC & $\mathrm{mgL}^{-1}$ & $4.4 \pm 2$ \\
\hline SST & $\mathrm{mgL}^{-1}$ & $4 \pm 2$ \\
\hline $\mathrm{BOD}_{5}$ & $\mathrm{mgL}^{-1}$ & $4 \pm 1$ \\
\hline COD & $\mathrm{mgL}^{-1}$ & $10.5 \pm 3$ \\
\hline$U V_{228}$ & $\mathrm{~m}^{-1}$ & 59.06 \\
\hline$U_{254}$ & $\mathrm{~m}^{-1}$ & 9.97 \\
\hline$U_{276}$ & $m^{-1}$ & 8.26 \\
\hline $\mathrm{UV}_{280}$ & $\mathrm{~m}^{-1}$ & 7.92 \\
\hline$U V_{282}$ & $m^{-1}$ & 7.75 \\
\hline $\mathrm{UV}_{284}$ & $\mathrm{~m}^{-1}$ & 7.59 \\
\hline
\end{tabular}

Figure 3: Caratterizzazione delle acque reflue prima della contaminazione farmaceutica 